\title{
Vacuum Assisted-Biopsy and Mammo-Guided Localization and Excisional Biopsy in the Microcalcifications Sampling of Breast: An Experience of Single Institution
}

\author{
Minho Park, ${ }^{1}$ and Yu-Mee Sohn ${ }^{1, *}$ \\ ${ }^{1}$ Department of Radiology, Kyung Hee University Hospital, College of Medicine, Kyung Hee University, Seoul, Korea \\ "Corresponding author: Yu-Mee Sohn, Department of Radiology, Kyung Hee University Hospital, College of Medicine, Kyung Hee University, Seoul, Korea. Tel: +82-29588625, Fax: \\ +82-29680787, E-mail: sonyumee@naver.com
}

Received 2015 June 05; Revised 2015 October 13; Accepted 2015 November 17.

\begin{abstract}
Background: Sterotactic vacuum assisted biopsy (ST-VAB) is safe and effective method for nonpalpable mammographically visible microcalcifications or masses. ST-VAB is less invasive and is associated with less scar formation than surgical excision. Also ST-VAB can avoid unnecessary additional surgery. But ST-VAB has possibility of histologic underestimation of high-risk lesions such as atypical ductal hyperplasia and atypical lobular hyperplasia.

Objectives: To evaluate the outcome of ST-VAB and mammography-guided localization and excisional biopsy (MGL-EB) for microcalcifications.

Patients and Methods: Two radiologists retrospectively reviewed the medical records of patients who underwent breast biopsy for microcalcification from January 2011 to March 2013. They underwent ST-VAB $(n=22)$ and MGL-EB $(n=34)$. The clinicoradiological factors of two groups were evaluated, respectively. The malignancy rate and imaging histologic discordant rate in the two groups were assessed. We evaluated follow-up studies of all patients for newly developed or missed breast cancer.

Results: The malignancy rates were 13.6\% (3/22) for ST-VAB and 17.6\% (6/34) for MGL-EB, respectively. Subsequent surgery was performed in five patients $(n=3, S T-V A B ; n=2, M G L-E B)$ and they were all confirmed as ductal carcinoma in situ. The discordant rates were $22.7 \%$ (5/22) for ST-VAB and $14.7 \%$ (5/34) for MGL-EB after imaging-histologic correlation. There was no malignancy detected on follow up studies.

Conclusion: ST-VAB and MGL-EB are reliable biopsy methods for microcalcifications. In proper indications, breast microcalcifications could be obtained by each method without missing diagnosis of breast cancer.
\end{abstract}

Keywords: Mammography, Biopsy, Image-Guided Biopsy, Breast Neoplasms, Breast Diseases

\section{Background}

Since 1970, the indications for use of stereotactic vacuum assisted biopsy (ST-VAB) have expanded to biopsy for pathological confirmation and benign mass removal. In particular, ST-VAB is a safe and effective method for nonpalpable mammographically visible microcalcifications or masses, and can yield large amounts of tissue (1-5). Furthermore, a lower re-biopsy rate and a fewer cases of histologic underestimation of ST-VAB with this method compared to core needle biopsy (CNB) were reported (5). However, highrisk lesions such as atypical ductal hyperplasia (ADH), and atypical lobular hyperplasia (ALH) can be underestimated even with sampling by ST-VAB. Thus, some groups support surgical excision is still supported as the gold standard (6).

Based on the imaging-histological discordance of ultrasound (US)-guided core needle biopsy results, recent studies indicated that US-guided vacuum-assisted excision can replace surgical excision $(7,8)$. With regard to microcalcifications, ST-VAB is less invasive and is associated with less scar formation than surgical excision (9). In addition, unnecessary surgery can be avoided (10).

\section{Objectives}

In this paper, we intended to evaluate the efficiency and investigate the association of clinicoradiological factors of ST-VAB and mammography-guided localization and excisional biopsy (MGL-EB) for sampling of suspicious microcalcifications.

\section{Patients and Methods}

\subsection{Study Population}

The institutional review board (IRB) of our institution approved this retrospective study and the requirement for informed consent was waived. The study population consisted of 56 patients who underwent mammography and biopsies for microcalcifications between January 2011 and 
May 2013. Two radiologists retrospectively reviewed the imaging findings, medical records, and pathology reports. Clinical variables such as age, symptoms, indications for mammography, family history, and personal medical history were reviewed based on medical records. Symptoms were classified as palpable lesion, pain, discomfort, nipple discharge, and axillary mass. The indication for mammography was divided as screening or diagnostic. Patients' personal histories of breast cancer, family history, and previous breast biopsy history were also investigated.

\subsection{Image Interpretation}

Mammograms were obtained with dedicated equipment (Senographe DS; GE Healthcare, Milwaukee, WI). Routine craniocaudal (CC) and mediolateral oblique (MLO) views were obtained. Additional views, such as spot magnification view, were also obtained if necessary. Breast parenchymal density was categorized by the breast imaging reporting and data system (BI-RADS) (11). The maximal extent of the removed microcalcifications was measured. The mammographic findings were categorized according to the BI-RADS assessment criteria. One dedicated breast radiologist with 8 years of experience (S.Y-M.) and one second-grade resident (P.M.) reviewed imaging-histological discordance after review of images and pathology reports. When the histological findings provided an explanation for the imaging features, they were defined as concordant, and otherwise defined as discordant (7) (Figure 1). Discordant lesions included the followings; 1 , lesions that were suspicious for malignancy and in which the pathologic findings did not compatible to imaging findings; 2 , lesions that were highly susggestive of malignancy, revealing benign pathologic results; or 3, no identified microcalcifications on specimen mammography, pathologic results, or both (7) (Figure 2). The malignant rates, defined that the number of malignant lesions divided by total biopsy cases at each group, were also evaluated. In cases with high-risk lesions [ADH, ALH, lobular carcinoma in situ (LCIS), radial scar, papillary lesion, etc.] and in which malignant pathology results were obtained by ST-VAB or MGLEB, subsequent therapeutic surgery was performed. After surgery, the pathological results were also reviewed.

\subsection{Biopsy Technique}

Biopsy of sonographically undetectable microcalcifications was performed. The biopsy method was determined according to the preference of the physician or patient after the radiologists' recommendation. Radiologist recommendation was done according to the location of microcalcification. For microcalcification located close to the chest wall or nipple, localization was more commonly recommended rather than stereotactic vacuum assisted biopsy. After obtaining the patient's informed consent, all procedures were performed by one dedicated breast radiologist with 8 years of experience in breast imaging.

Twenty-two patients underwent ST-VAB, which was performed with a vacuum-assisted device (Mammotome; Ethicon Endosurgery, Cincinnati, $\mathrm{OH}$ ) in the decubitus position using a Decubitis Breast Imaging table. According to the extent of microcalcifications, 12 patients were underwent ST-VAB using an 8-gauge probe $(>10 \mathrm{~mm}$ extent), and 10 underwent ST-VAB using 11-gauge probe $(<10$ mm extent). After biopsy, microcalcification sampling was confirmed by specimen mammography. When the procedure was completed, after removal of the stereotactic probe, we checked for complications, such as bleeding and hematoma, and compression adequate to avoid bleeding or hematoma was performed (Figure 1). After one week after ST-VAB, patients were scheduled to follow up spot magnification view. Thirty-four patients underwent MGL-EB. Kopans needles $5 \mathrm{~cm}(\mathrm{n}=4), 7.5 \mathrm{~cm}(\mathrm{n}=27)$, or $10 \mathrm{~cm}$ $(\mathrm{n}=3)$ in size (Cook, Bloomington, IN) were used according to the location of microcalcifications. Mammographyguided needle insertion was performed. The needle was inserted to the optimal position by placing microcalcifications within the reinforcement segment (12). If clustered microcalcifications were present as long extent, two wires were used for bracket localization (1). After excision, adequate sample retrieval was confirmed by taking specimen mammography (Figure 3) and follow up spot magnification view was taken one week after procedure and on benign lesions, follow-up mammography and spot magnification views were performed at least two years.

\section{Results}

The median ages of patients were 50.5 years (range, 28 - 63 years) for ST-VAB and 51 years (range, 30 - 69 years) for MGL-EB. None of the patients had clinical symptoms, such as palpable mass, pain, or nipple discharge. There were no patients with a family history of breast cancer. With regard to the personal history of breast cancer, four patients had prior experience of breast surgery; all underwent MGL-EB.

The indications for mammography were screening in 8 cases and diagnostic purposes in 14 cases among the patients undergoing ST-VAB $(\mathrm{n}=22)$, and screening in 10 cases and diagnostic in 24 cases among those undergoing MGL$\mathrm{EB}(\mathrm{n}=34)$. The diagnostic mammography were performed due to interval change of microcalcifications on follow up mammography $(\mathrm{n}=29)$ and abnormal finding on mammography taken from other hospitals $(n=9)$.

Table 1 shows the clinical and radiological characteristics of the two biopsy methods. There was no inadequate 

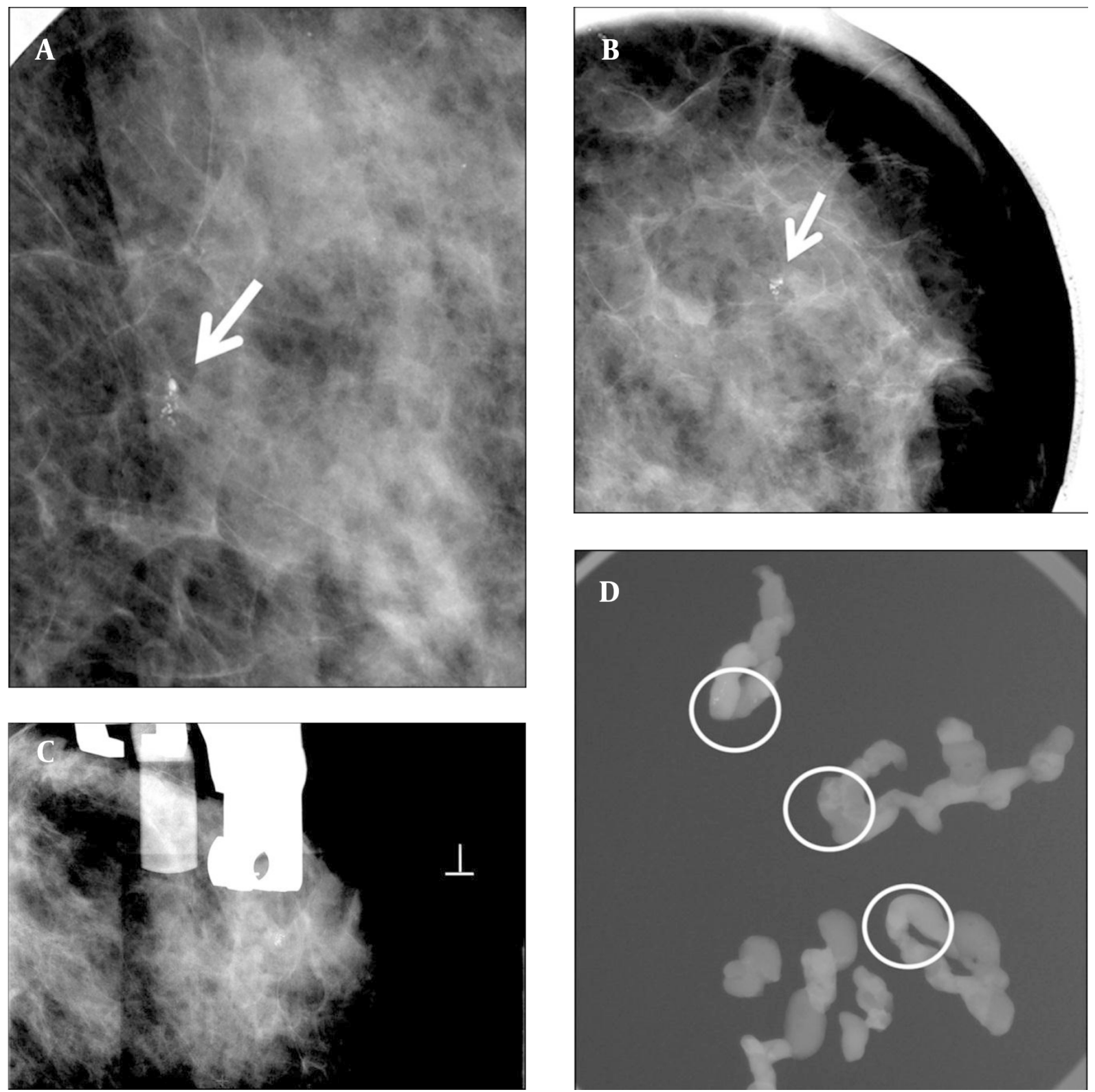

Figure 1. A 55-year-old woman with abnormal left mammogram categorized as 4a. A and B, Left craniocaudal magnification and mediolateral oblique magnification view showed suspicious clustered microcalcifications in the outer breast (arrow). C, Stereotactic biopsy was done. D, Specimen radiography of some samples from an 8 gauge biopsy showed multiple microcalcifications (circled). Pathology of biopsy revealed ductal carcinoma in situ. The imaging and histologic finding was considered concordant. Subsequent surgery was done. Pathologist confirmed no residual malignancy.

sampling in each method. Follow up spot magnification view taken one week after procedure showed microcalcification on the area of interest was correctly sampled.

The malignancy rates were $13.5 \%$ [3/22, 3 ductal carcinoma in situ (DCIS)] in ST-VAB and 17.6\% [6/34, 5 DCIS and 1 invasive ductal carcinoma (IDC)] in MGL-EB(Table 2). Breast conserving surgery (BCS) was done for malignancies in the
ST-VAB group. Follow-up studies were performed in these patients in the ST-VAB group at least 2 years. There was no delayed malignancy occurrence. In the MGL-EB group, after the pathology identified, two patients went to another hospital. Other four patients had additional radiation therapy. Follow-up studies were performed at least 2 years. In one patient, new DCIS was found at contralateral 

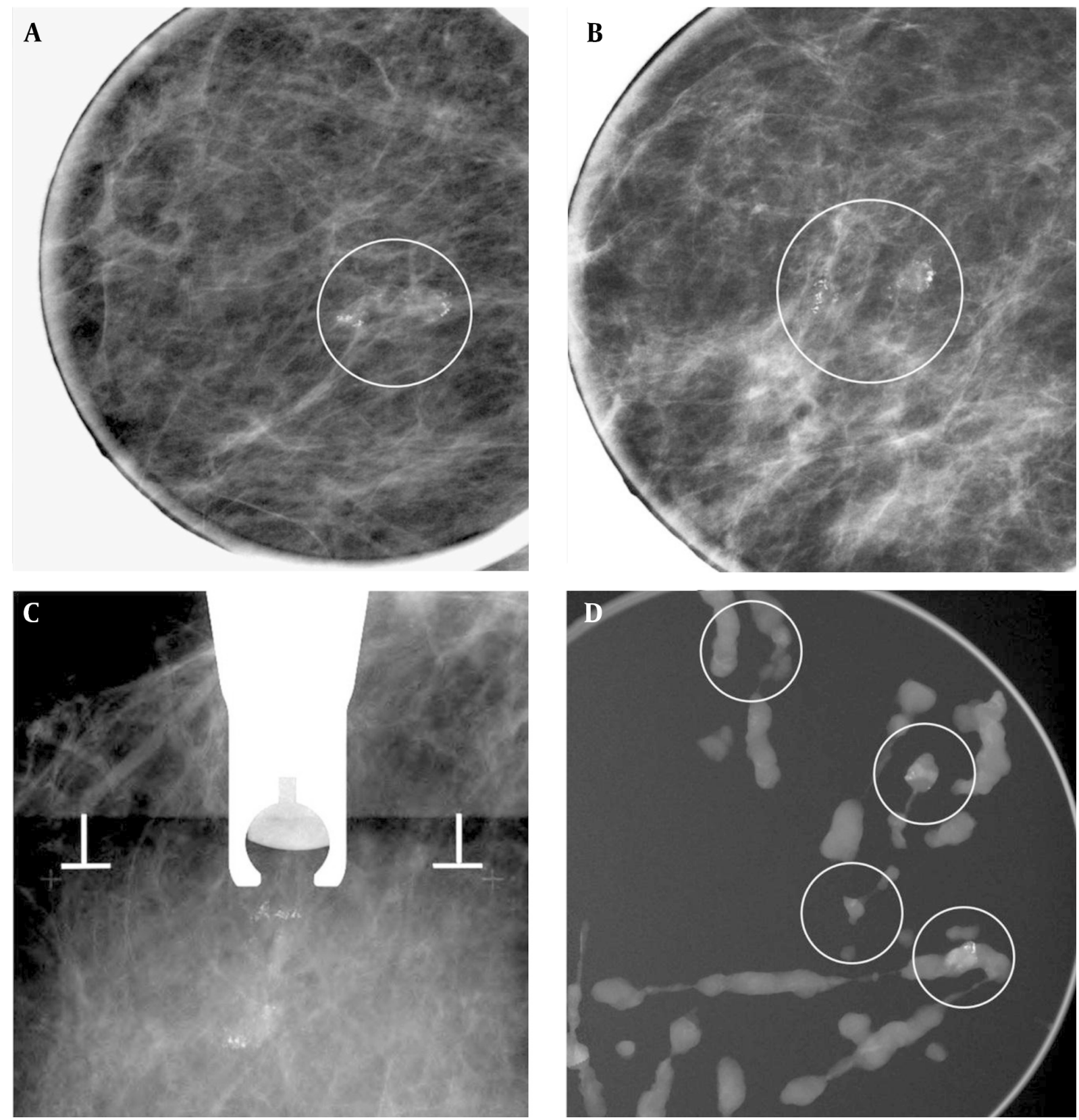

Figure 2. A 62-year-old woman with right mammogram categorized as $4 \mathrm{~b}$. A and B, Right craniocaudal magnificantion and mediolateral oblique magnificantion view showed suspicious clustered microcalcifiecation (circled). C, Stereotatic biopsy was done. D, Specimen radiography of some samples from an 8 gauge biopsy showed enough sampling of microcalfication (circled). Pathology of biopsy revealed fibrocystic disease with microcalcifications. Imaging-histologic correlation was considered discordant. Subsequent surgery was planned, but follow-up mammography was performed due to patient's refuse. There was no interval change occurred on follow up mammography and spot magnification view at least 2 years and regarded as benign.

breast. On follow-up studies for remaining three patients, there was no cancer recurrence.

There was no delayed malignancy in all patients with benign breast lesions at least 2 years.

Imaging-histological discordance rates were $22.7 \%$
(5/22) for ST-VAB and 14.7\% (5/34) for MGL-EB after radiologist's review. One discordant case in the ST-VAB group was BI-RADS category $4 \mathrm{~b}$ lesion. Other discordant cases were BI-RADS category 4a lesions. On final pathologic reports, first case was confirmed as pseudocyst and fibrosis. Sec- 

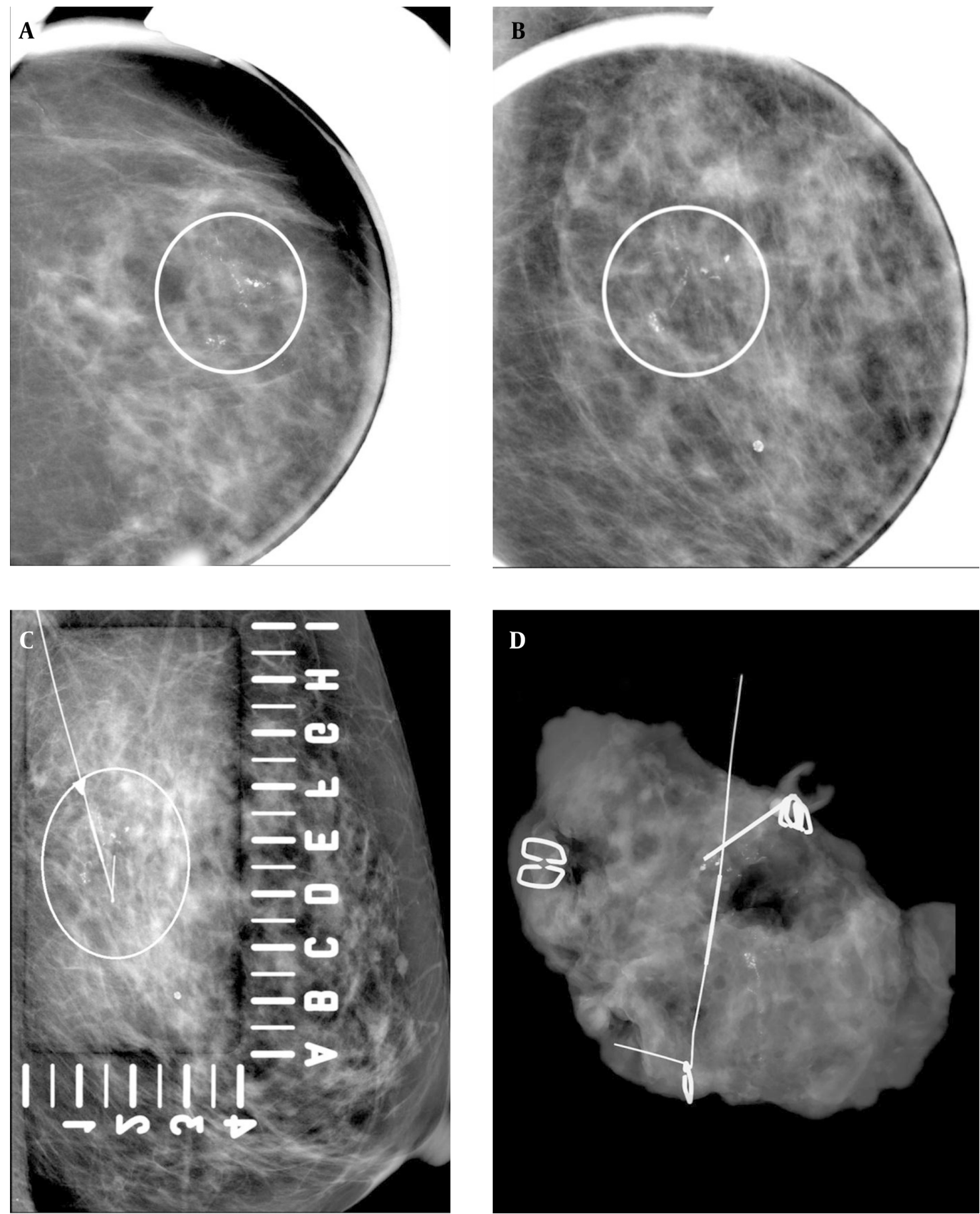

Figure 3. A 69-year-old woman with abnormal left mammogram categorized as 4c. A and B, Craniocaudal magnification and mediolateral oblique magnification view showed suspicious segmental fine linear and branching microcalcifications (circled). C, Mammography-guided needle localization was performed (circled). D, Specimen radiography of surgical specimen revealed adequate sampling of microcalcifications. Pathology of biopsy revealed DCIS. The imaging and histologic finding was considered concordant.

ond case was diagnosed as fibrocystic change. Subsequent surgery for imaging-histologic discordant case was not performed because of surgeon's decision and patient's refuse. Follow-up studies were performed in three patients 
Table 1. Clinicoradiologic Differences in ST-VAB and L-EB ${ }^{\mathrm{a}}$

\begin{tabular}{|c|c|c|c|}
\hline & & ST-VAB $(n=22)$ & $\mathbf{L}-\mathbf{E B}(\mathbf{n}=\mathbf{3 4})$ \\
\hline Age, year & & $46.70 \pm 8.78$ (Range $28-63)$ & $50.51 \pm 10.49$ (Range $30-69)$ \\
\hline \multirow{2}{*}{ Indication } & S & 8 & 10 \\
\hline & $\mathrm{D}$ & 14 & 24 \\
\hline Lesion size, cm & & $8.49 \pm 5.39$ (Range $1.5-22$ ) & $5.72 \pm 6.71($ Range $1.4-21)$ \\
\hline \multirow{3}{*}{ MMG-pattern } & 2 & $2(9.09)$ & $2(5.88)$ \\
\hline & 3 & $15(68.22)$ & $17(50.00)$ \\
\hline & 4 & $4(18.18)$ & $15(44.12)$ \\
\hline \multirow{4}{*}{ BI-RADS } & 3 & $3(13.64)$ & $1(2.94)$ \\
\hline & $4 a$ & $17(77.27)$ & $32(94.12)$ \\
\hline & $4 \mathrm{~b}$ & $2(9.09)$ & 0 \\
\hline & $4 \mathrm{C}$ & 0 & $1(2.94)$ \\
\hline
\end{tabular}

Abbreviations: D, diagnostic exam; MMG, mammography; S, screening exam; ST-VAB, stereotactic vacuum assisted biopsy; L-EB, localization and excisional biopsy; BIRADS, breast imaging reporting and data system.

${ }^{\mathrm{a}}$ Table numbers are No.(\%) except for mean age and mean size.

Table 2. Histologic Results of Two Biopsy Methods

\begin{tabular}{|c|c|c|c|c|}
\hline \multirow[t]{2}{*}{ Biopsy } & \multicolumn{2}{|c|}{ ST-VAB } & \multicolumn{2}{|r|}{ L-EB } \\
\hline & Number of Cases & Histologic Results & Number of Cases & Histologic Results \\
\hline Malignant lesions & 3 & 3 DCIS & 6 & 5 DCIS; 1 IDC \\
\hline High risk lesion & 1 & 1 sclerosing adenosis & 6 & $\begin{array}{c}1 \text { LCIS } 1 \text { sclerosing adenosis; } 1 \text { Atypical ductal hyperplasia; } 3 \text { Intraductal } \\
\text { papilloma }\end{array}$ \\
\hline Benign lesion & 18 & 18 fibrocystic change & 22 & 21 Fibrocystic change; 1 Pseudocyst and fibrosis \\
\hline Total & 22 & & 34 & \\
\hline
\end{tabular}

in the ST-VAB group (mean 27.3, range 25 - 31 months) and five patients in the MGLEB group (mean 24.6, range 23 27 months). There was no malignancy detected on follow up studies. No significant biopsy-related complication occurred in any of the patients in each method.

\section{Discussion}

Many biopsy methods are available for breast microcalcifications. Mammography-guided biopsy is necessary for sonographically undetected microcalcifications. One of the mammography-guided biopsy methods is ST-VAB. It is effective for non-palpable, mammographically visible microcalcifications or masses (13).

Kettritz et al. (14) reported that the malignancy rate is $58 \%$ in ST-VAB for suspicious calcification. Luparia et al. (4) reported malignancy rates of microcalcifications categorized by BI-RADS category equal to $7.4 \%$ in category 3, 25.1\% in category 4 , and $100 \%$ in category 5 . In other previous studies, the malignancy rate was $0-19 \%$ in category 3 , and $15.3-36.6 \%$ in category $4(4,15,16)$. In our study, the malignancy rates were $6 / 32(18.8 \%)$ in category 4 , and zero in category 3 . These are in accordance with those of previous studies.

In the prospective multicenter COBRA study (core biopsy after radiological localisation), Verkooijen et al. showed that stereotactic large-core needle biopsy of nonpalpable breast disease has high diagnostic efficacy, with a sensitivity rate of $97 \%$ and specificity rate of $99 \%$, and can safely replace needle-localized open breast biopsy (17). Liberman et al. (18) suggested that the number of operations can be reduced by ST-VAB. In addition, there is a greater likelihood of sparing a surgical procedure and 
saving cost using an 11-gauge rather than a 14-gauge needle. Some previous reports indicated that ST-VAB could yield excellent results with good diagnostic efficacy, safety, and cost-effectiveness, when adequate indications are prepared.

However, when selecting biopsy methods, it is necessary to consider the possible contraindications. In ST-VAB, if the microcalcifications are too faint to detect, ST-VAB were difficult to be performed. In addition, if the location of lesion was deep, near chest wall, or too close from skin, there is a risk of causing chest wall or skin injury. Other biopsy methods, such as excisional biopsy, should be considered in such cases (13). Additionally, Common drawback of stereotactic biopsy is underestimation. It means that the high risk lesion could be incorrectly diagnosed by insufficient biopsy and correctly diagnosed as malignancy by subsequent surgery. In this study, because the number of patients undergoing subsequent surgery was too small, it was not possible to evaluate underestimation rate. Previously reported underestimation rates were 10 - 27\% in ADH, and $4-18 \%$ in DCIS (19). The underestimation rate can vary according to needle size, with underestimation rates of 0 - 38\% with an 11-gauge VAB needle and higher rates with a 14-gauge VAB needle (19). Use of an 8-gauge needle can improve diagnostic performance in comparison with an 11gauge needle (4). However, increasing the needle size is associated with greater risk of bleeding and hematoma formation, so it is necessary to select the needle size appropriate for each application (20). In this study, an 8-gauge needle was used in cases in which the extent of microcalcifications was $>10 \mathrm{~mm}$, while an 11-gauge needle was used in those with less extensive microcalcifications $<10 \mathrm{~mm}$. There were no complications by ST-VAB.

MGL-EB has been used instead of ST-VAB when the location of microcalcification was not suitable for ST-VAB for non-palpable breast lesions, not only calcification but also masses and calcified masses. There is a high degree of accuracy at biopsy, but this method has limitations. Most of all, surgery can place a large burden on patients. The morbidity, mortality and the cost in surgery have been discussed as disadvantage of surgery in previous study and wire localization is necessary for non-palpable lesions $(3,21)$. If the wire inadvertently dislodges, migrates or is transected, the surgeon can become disoriented and excise the wrong tissue (22). When malignancy is diagnosed, the positive margin has a broad range of $26-84 \%$. In such cases, a subsequent second operation is needed, which can increase the morbidity and the mortality rates (21).

For imaging-histologic discordant lesions, re-biopsy or surgical excision has been recommended to obtain a definite diagnosis (7). In stereotactic biopsy, the reported imaging-histologic discordance rate ranges from 3.1 to
6.2\%. When the subsequent procedure (surgical excision or repeat biopsy) has been performed at the discordant lesion after stereotactic biopsy, the frequency of malignancy ranged from 0 to $64 \%(7,20,23-25)$. In this study, imaging histologic discordance of ST-VAB is $22.7 \%$. It is relatively higher than previous study's results. This might be that the authors tried to reach an interpretation consensus between two radiologists on the evaluation of imaginghistologic discordant lesions if there was a discrepancy between two radiologists.

In conclusion, according to the results of our series, both ST-VAB and MGL-EB are very effective and safe procedure for sampling of microcalcification. Under proper indication, each method can reduce the benign biopsy and diagnose exactly. And also, breast microcalcifications could be correctly obtained by each method without missing breast cancer.

\section{Footnotes}

Authors' Contribution: The manuscript was resulted from Yu-Mee Sohn and Minho Park; the supervisor professors was Yu-Mee Sohn.

Financial Disclosure: There was no conflict of interest.

\section{References}

1. Liberman L, Kaplan J, Van Zee KJ, Morris EA, LaTrenta LR, Abramson $\mathrm{AF}$, et al. Bracketing wires for preoperative breast needle localization. AJR Am J Roentgenol. 2001;177(3):565-72. doi: 10.2214/ajr.177.3.1770565. [PubMed: 11517048]

2. Tonegutti M, Girardi V. Stereotactic vacuum-assisted breast biopsy in 268 nonpalpable lesions. Radiol Med. 2008;113(1):65-75. doi: 10.1007/s11547-008-0226-0. [PubMed: 18338128].

3. Masroor I, Afzal S, Shafqat G, Khattak YJ. Comparison of stereotactic core breast biopsy and open surgical biopsy results at a tertiary care hospital in Pakistan. Int J Womens Health. 2011;3:193-6. doi: 10.2147/IJWH.S22853. [PubMed: 21792341].

4. Luparia A, Durando M, Campanino P, Regini E, Lucarelli D, Talenti A, et al. Efficacy and cost-effectiveness of stereotactic vacuum-assisted core biopsy of nonpalpable breast lesions: analysis of 602 biopsies performed over 5 years. Radiol Med. 2011;116(3):477-88. doi:10.1007/s11547011-0625-x. [PubMed: 21225359].

5. Venkataraman S, Dialani V, Gilmore HL, Mehta TS. Stereotactic core biopsy: Comparison of 11 gauge with 8 gauge vacuum assisted breast biopsy. Eur J Radiol. 2012;81(10):2613-9. doi: 10.1016/j.ejrad.2011.10.027. [PubMed: 22127375].

6. Gisvold JJ, Goellner JR, Grant CS, Donohue JH, Sykes MW, Karsell PR, et al. Breast biopsy: a comparative study of stereotaxically guided core and excisional techniques. AJR Am J Roentgenol. 1994;162(4):815-20. doi: 10.2214/ajr.162.4.8140997. [PubMed: 8140997].

7. Kim MJ, Kim EK, Lee JY, Youk JH, Park BW, Kim SI, et al. Breast lesions with imaging-histologic discordance during US-guided $14 \mathrm{G}$ automated core biopsy: can the directional vacuum-assisted removal replace the surgical excision? Initial findings. Eur Radiol. 2007;17(9):2376-83. doi: 10.1007/s00330-007-0603-4. [PubMed: $17361422]$ 
8. Wang ZL, Liu G, Li JL, Su L, Liu XJ, Wang W, et al. Breast lesions with imaging-histologic discordance during 16-gauge core needle biopsy system: would vacuum-assisted removal get significantly more definitive histologic diagnosis than vacuum-assisted biopsy? Breast J. 2011;17(5):456-61. doi: 10.1111/j.1524-4741.2011.01128.x. [PubMed: 21762244].

9. Hillner BE. Financial costs, benefits, and patient risk preferences in node-negative breast cancer: insights from a decision analysis model. Recent Results Cancer Res. 1993;127:277-84. [PubMed: 8502826].

10. Liberman L, LaTrenta LR, Van Zee KJ, Morris EA, Abramson AF, Dershaw DD. Stereotactic core biopsy of calcifications highly suggestive of malignancy. Radiology. 1997;203(3):673-7. doi: 10.1148/radiology.203.3.9169687. [PubMed: 9169687].

11. American college of radiology . Breast imaging reporting and data system (bi-rads): Mammography. 4 ed. ; 2003.

12. Sanders LM, Kalisher L, Trikha S. Needle localization using a stereotactic table: a reassessment. AJR Am J Roentgenol. 2000;174(6):1689-90. doi: 10.2214/ajr.174.6.1741689. [PubMed: 10845507].

13. Faour I, Al-Salam S, El-Terifi $\mathrm{H}$, El Taji $\mathrm{H}$. The use of a vacuum-assisted biopsy device (Mammotome) in the early detection of breast cancer in the United Arab Emirates. Ann N Y Acad Sci. 2008;1138:108-13. doi: 10.1196/annals.1414.016. [PubMed: 18837890].

14. Kettritz U, Morack G, Decker T. Stereotactic vacuum-assisted breast biopsies in 500 women with microcalcifications: radiological and pathological correlations. Eur J Radiol. 2005;55(2):270-6. doi: 10.1016/j.ejrad.2004.10.014. [PubMed: 16036159].

15. Mendez A, Cabanillas F, Echenique M, Malekshamran K, Perez I, Ramos E. Evaluation of Breast Imaging Reporting and Data System Category 3 mammograms and the use of stereotactic vacuumassisted breast biopsy in a nonacademic community practice. Cancer. 2004;100(4):710-4. doi: 10.1002/cncr.20017. [PubMed: 14770425].

16. Travade A, Isnard A, Bagard C, Bouchet F, Chouzet S, Gaillot A, et al. Stereotactic 11-gauge directional vacuum-assisted breast biopsy: experience with 249 patients. J radiologie. 2002;83(9 Pt 1):1063-71.

17. Verkooijen HM, Core Biopsy After Radiological Localisation Study G.
Diagnostic accuracy of stereotactic large-core needle biopsy for nonpalpable breast disease: results of a multicenter prospective study with 95\% surgical confirmation. Int J Cancer. 2002;99(6):853-9. doi 10.1002/ijc.10419. [PubMed: 12115488].

18. Liberman L, Dershaw DD, Rosen PP, Abramson AF, Deutch BM, Hann LE. Stereotaxic 14-gauge breast biopsy: how many core biopsy specimens are needed?. Radiology. 1994;192(3):793-5. doi: 10.1148/radiology.192.3.8058949. [PubMed: 8058949]

19. Dhillon MS, Bradley SA, England DW. Mammotome biopsy: impact on preoperative diagnosis rate. Clin Radiol. 2006;61(3):276-81. doi: 10.1016/j.crad.2005.08.017. [PubMed: 16488210].

20. Schaefer FK, Order BM, Eckmann-Scholz C, Strauss A, Hilpert $\mathrm{F}$, Kroj $\mathrm{K}$, et al. Interventional bleeding, hematoma and scarformation after vacuum-biopsy under stereotactic guidance: Mammotome((R))-system $11 \mathrm{~g} / 8 \mathrm{~g}$ vs. ATEC((R))-system $12 \mathrm{~g} / 9 \mathrm{~g}$. Eur J Radiol. 2012;81(5):e739-45. doi: 10.1016/j.ejrad.2012.01.033. [PubMed: 22381441].

21. Dogan L, Gulcelik MA, Yuksel M, Uyar O, Reis E. Wire-guided localization biopsy to determine surgical margin status in patients with non-palpable suspicious breast lesions. Asian Pac J Cancer Prev 2012;13(10):4989-92. [PubMed: 23244096].

22. Schnall MD, Orel SG, Connick TJ. MR guided biopsy of the breast. Magn Reson Imaging Clin NAm. 1994;2(4):585-9. [PubMed: 7489310].

23. Jackman RJ, Nowels KW, Shepard MJ, Finkelstein SI, Marzoni FJ. Stereotaxic large-core needle biopsy of 450 nonpalpable breast lesions with surgical correlation in lesions with cancer or atypical hyperplasia. Radiology. 1994;193(1):91-5. doi: 10.1148/radiology.193.1.8090927. [PubMed: 8090927].

24. Bauer M, Schulz-Wendtland R, Krämer S, Buhner M, Lang N, Tulusan $\mathrm{AH}$. [Indications, technique and results of ultrasound guided punch biopsy in breast diagnosis $(\mathrm{n}=307)]$. Geburtshilfe und Frauenheilkunde. 1994;54(10):539-44.

25. Liberman L, Drotman M, Morris EA, LaTrenta LR, Abramson AF, Zakowski MF, et al. Imaging-histologic discordance at percutaneous breast biopsy. Cancer. 2000;89(12):2538-46. 